\title{
TTR
}

Traduction, terminologie, re?daction

\section{Néologie lexicale : transfert, adaptation, innovation}

\section{Silvia Pavel}

Volume 2, numéro 1, 1er semestre 1989

Carrefours de la traduction

URI : https://id.erudit.org/iderudit/037038ar

DOI : https://doi.org/10.7202/037038ar

Aller au sommaire du numéro

Éditeur(s)

Association canadienne de traductologie

ISSN

0835-8443 (imprimé)

1708-2188 (numérique)

Découvrir la revue

Citer cet article

Pavel, S. (1989). Néologie lexicale : transfert, adaptation, innovation. TTR, 2(1),

125-137. https://doi.org/10.7202/037038ar

Tous droits réservés @ TTR: traduction, terminologie, rédaction — Les auteurs, d'utilisation que vous pouvez consulter en ligne.

https://apropos.erudit.org/fr/usagers/politique-dutilisation/ 


\section{Néologie lexicale: transfert, adaptation, innovation}

\section{Silvia Pavel}

Il est, en matière de néologie, des évidences qu'il importe parfois de rappeler. L'une d'elles est la dimension «théorétique» du langage humain: il peut être décrit comme un système de signes dont l'agencement régi par des règles sert à la représentation discursive de la «nature des choses», à la conceptualisation des perceptions du monde réel, à l'anticipation de mondes possibles. Une autre est la dimension sociale du langage: il sert également à la communication entre les individus d'une communauté. C'est un acte de création sociale, d'orientation du comportement individuel, continuellement régénéré dans l'interaction collective à l'intérieur d'espaces historiquement et culturellement déterminés.

Les rapports entre pensée et langage occupent de plus en plus le devant de la scène contemporaine:

La linguisticisation de la pensée peut être interprétée comme le prélude, la préparation à une matérialisation infiniment plus radicale dont l'avènement a fait bien plus que commencer. L'informatisation, la multiplication de toutes les technolangues, le développement de l'intelligence artificielle et des sciences cognitives, les linguistiques génératives fondées sur des épistémologies de la simulation, les modélisations opératoires de l'intentionnalité et de la prise de décision, les programmes de traduction automatique, etc., avec toute la rhétorique qui les entoure et qui fait peu à peu basculer du côté de la technique le lexique des significations traditionnelles (pensée, intelligence, langage, dialogue, etc.), constituent quelques facettes de ce processus. (G. Hottois, 1987, pp. 66-67)

En même temps, l'évolution de la pensée (découvertes scientifiques, inventions techniques) est intimement liée à l'idée de progrès social et de changement linguistique:

As the use of new technology changes human practices, our ways of speaking about that technology change our language and our understanding. This new way of speaking in turn creates change in the world 
we construct... The development of the (computer) technology has led to new uses of terms such as «information», «input», «output», «language", and "communication", while work in areas such as artificial intelligence is bringing new meanings to words like «intelligence», "decision", and «knowledge». The technical jargon gives shape to our commonsense understanding in a way that changes our lives. (T. Winograd, 1986, p. 6)

En outre, le renouvellement du langage ne se limite pas à l'émergence de sens nouveaux dans la coquille des mots en circulation mais se manifeste à tous les niveaux du système linguistique. L. Guilbert (1975), par exemple, considère quatre aspects du changement lexical: la néologie phonologique, la néologie syntagmatique, la néologie sémantique et la néologie d'emprunt.

La création d'un mot et, à plus forte raison, son évolution, sa productivité peuvent relever de plusieurs procédés néologiques. Ainsi le mot «néologisme», attesté pour la première fois en 1735 dans une fable allégorique, est un exemple de néologie syntagmatique (dérivation, composition) par combinaison des éléments «neos» et «logos» empruntés au grec (néologie d'emprunt). Ses sens premiers étaient péjoratifs: vice du style, tour affecté des phrases, jonction téméraire des mots, bizarrerie, fadeur et petitesse des figures, expression nouvelle et éloignée de celles que l'usage autorise, etc. (F. Dougnac, 1982). Les Précieuses du XVII ${ }^{e}$ siècle ridiculisées sans merci par Molière en illustrèrent avant la lettre l'abus élitiste. Selon le Petit Robert (1988), l'adjectif «néologique» (1726) précède «néologisme» d'une dizaine d'années avec une acception non moins péjorative, mais «néologie» (1759) a déjà un sens neutre sinon mélioratif: «emploi de mots nouveaux dans la langue afin de l'enrichir". Le sens moderne de "néologisme» remonte à 1800: "Emploi d'un mot nouveau soit créé, soit obtenu par déformation, dérivation, composition, emprunt, etc., ou emploi d'un mot dans un sens nouveau (néologisme de sens).» Pris dans cette acception, «néologisme» est lui-même un exemple de néologie sémantique. Le sens le plus récent est aussi un sens spécialisé: en psychiatrie, le terme «néologisme» désigne «un mot forgé par un malade mental, incompréhensible par l'entourage». C'est un exemple de néologie sémantique au deuxième degré. En littérature, Orwell illustre par la «novlangue» une autre forme d'aliénation, collective celle-là.

L'étude des changements formels et sémantiques accorde une attention croissante à la combinatoire sociolinguistique de leurs manifestations statiques/simultanées et dynamiques/évolutives. Lexicographes et linguistes reconnaissent d'ailleurs volontiers le caractère théoriquement distinct mais pratiquement indissociable de ces manifestations (A. Rey, 1976, pp. 3-17). Ainsi, la dénomination d'un concept nouveau relève de facteurs socio-culturels tels l'universalité, la transparence symbolique, les qualités mnémotechniques, la créativité et le prestige 
individuels, qui déterminent l'acceptabilité du néologisme dans un groupe social.

Le phénomène des concepts nomades (I. Stengers, 1987) impossible à ignorer dans les activités technoscientifiques - souligne la dimension sociale du sentiment de nouveauté: des termes usuels dans un secteur d'activité peuvent migrer vers une spécialité émergente et y faire figure de néologismes, qu'ils soient ou non accompagnés d'un changement sémantique.

La nouveauté d'une forme obtenue par dérivation, composition, abréviation ou emprunt externe est déclenchée par la nouveauté sémantique, quand elle ne résulte pas tout simplement de la mobilité de l'ensemble lexical ou du besoin de renommer des concepts. D'autre part, la nouveauté sémantique - génératrice de néologismes formels - concerne également les néologismes de sens, les lexies complexes, les emplois analogiques et figurés. Elle se conçoit difficilement en dehors du fonctionnement concret du langage ou du renouvellement de la pensée.

L'observation un tant soit peu attentive de ces phénomènes confirme des intuitions communément partagées. On constate que le vocabulaire se renouvelle bien plus rapidement que la grammaire ou la prononciation et davantage dans les périodes d'effervescence intellectuelle: en pleine «révolution informatique» (1948-1960), un quart du lexique français avait subi des modifications (J. Thiele, 1987, p. Il) tandis que le système phonologique et la grammaire n'avaient été que marginalement touchés (J. Cellard, 1988). Sur le plan lexical, les besoins de dénomination des nouveautés technoscientifiques l'emportent largement sur les besoins expressifs des locuteurs dans leurs échanges quotidiens: en français courant, il suffit de 1500 à 3000 mots pour bien communiquer, tandis que le vocabulaire de l'informatique contient à lui seul plus de 10000 termes. Parmi les terminologies spécialisées, celles des secteurs de pointe se renouvellent le plus rapidement. C'està-dire que la nouvelle science du chaos (chaologie) et les mathématiques non linéaires, la physique des superconducteurs et ses retombées sur l'industrie électronique, le génie génétique et ses biotechnologies, ou l'intelligence artificielle et ses applications produisent aujourd'hui plus de néologismes que la sylviculture, la géologie ou l'industrie pétrolière dont le progrès dépend de l'innovation conceptuelle dans les secteurs dits de pointe. Il a fallu près d'un siècle pour que la terminologie du forage pétrolier change considérablement; l'intelligence artificielle n'existait pas il y a quarante ans, sa terminologie française a pour ainsi dire doublé depuis 1980 et l'on ne peut prédire ce qu'elle deviendra au cours des quinze prochaines années.

Cette massive entrée en scène des terminologies naissantes en spécialités de pointe suscite un renouveau d'intérêt pour la probléma- 
tique néologique. Intérêt pratique d'abord, parce que les besoins d'expression de la modernité technoscientifique exigent de les repérer en tant qu'entités produites, de les répertorier, de les décrire systématiquement et de les disséminer afin d'en généraliser l'usage, d'en faciliter la maîtrise et en même temps l'harmonisation. C'est là l'objet de la «néographie», nouvelle spécialité désignée par un néologisme syntagmatique. Intérêt théorique ensuite, parce que l'étude de ces néologismes nourrit l'actualité de la réflexion sur les rapports entre langue, communication et société. L'analyse du renouvellement lexical en tant que processus continu de transfert, d'adaptation et d'innovation fait l'objet de la «néologie», nouvelle spécialité désignée par un néologisme sémantique.

Considérons à titre d'exemple la langue d'une spécialité récente telle que l'intelligence artificielle - étude des comportements humains en vue de leur simulation informatique. La terminologie afférente désigne des concepts véhiculés, depuis 1956 et le plus souvent en anglais ${ }^{1}$, par une intelligentsIA internationale, hautement pluridisciplinaire (mathématiciens, logiciens, philosophes, linguistes, neurobiologistes, psychologues, informaticiens). Dans les milieux de l'IA, l'effervescence intellectuelle est entretenue autant par la rapidité et la diversité des échanges linguistiques (colloques, conférences, programmes de recherches et d'enseignement universitaires, salons, expositions, publications, courrier électronique) que par la véhémence des controverses entre diverses écoles de pensée et l'intensité de la concurrence technologique.

De son propre aveu, l'intelligentsIA se distingue par des méthodes de travail, par une vision du monde et des manières de l'exprimer qui lui sont propres, quelle que soit la langue choisie pour les véhiculer:

Each science is differentiated from others not merely by the set of phenomena it claims as its object of study, but also by the approach it takes (the science's view of those phenomena, its paradigm). If we view Man as an actor whose internal thought processes can't be investigated, then we are called «behavioral psychologists", and we study human behavior. If we view Man as a brain, as a piece of hardware built out of neurons, then we are called «biologists", and we study neurophysiological responses. If we view Man as a machine, as an automaton, then we're called "cyberneticists", and we investigate mathematical properties of feedback networks of simple components. If we adopt the view of Man as a processor of symbols, then we're working in the field of Artificial Intelligence. No one view of Man is «right» or «wrong»; each is adopted because from it we can build a model, which in turn has some practical consequences and uses. (D. Lenat, 1977, p. 259)

1. Pour un exposé éclairant sur la diffusion mondiale de l'anglo-américain, voir Claude Hagège, 1987, pp. 149-164. 
Comme tout vocabulaire spécialisé, le vocabulaire IA est perméable aux influences exercées par les sciences connexes et leurs manières de voir les objets étudiés:

... à l'arrière-plan de tous les stades de la recherche scientifique se projette une image théorique où se résume l'idiome dans lequel une génération d'esprits formule de préférence son appréhension du réel: mécanisme ou vitalisme, fixisme ou évolutionnisme, fonctionnalisme ou structuralisme, atomisme ou gestaltisme. Depuis l'essor de la biologie moléculaire, c'est le vocabulaire et la représentation des phénomènes empruntés à l'informatique et à la linguistique qui stylisent la sensibilité scientifique, laquelle s'exprime en termes de «programme», de "code» ou de «message». (J.-F. Revel, 1988, p. 167)

Compte tenu de ces repères sociolinguistiques, on peut tenter d'aborder le devenir du néologisme IA dans une dynamique lexicale inséparable de l'interaction sociale et circonscrite par la combinatoire d'une triple nécessité dénominationnelle: appropriation et transformation de l'univers conceptuel antérieur, originalité vis-à-vis du déjà-dit et partage social d'un espace de croyances, d'attitudes et de préférences langagières.

\section{Antériorité et transférabilité conceptuelles}

Le paysage conceptuel de cette nouvelle technoscience se dessine sur le fond de la pensée occidentale, par appropriation et modification graduelle de sous-ensembles conceptuels antérieurs autant que par création de concepts nouveaux. Des concepts traditionnels, tels que "cognition», "connaissance», «intention», «pensée», «apprentissage» subissent des restrictions ou des extensions de sens et reçoivent des définitions stipulatives parfois divergentes selon qu'ils sont appréhendés dans une perspective cognitiviste, connexionniste ou neurobiologique. Aussi l'interprétation neurobiologique de l'apprentissage diffère-t-elle considérablement de l'interprétation cognitiviste ou analytique:

Learning is not a process of accumulation of representations of the environment; it is a continuous process of transformation of behavior through continuous change in the capacity of the nervous system to synthesize it. (Maturana et Varela, 1980, p. 45)

Les multiples transferts du concept «résolution de problèmes» sont décrits par D. Andler (1987, p. 119) en ces termes:

Le concept... se propage d'abord au sein des sciences, passant de la science absolument formalisée - les mathématiques - aux savoirs les moins formalisés (l'histoire, par exemple, ou la philosophie); en même temps, de figure parmi d'autres de l'activité scientifique, le problème se transforme en figure exclusive ou du moins paradigmatique: cette «problématisation» des sciences est en partie une réalité, en partie un idéal méthodologique. Par ailleurs, le concept se propage, en se durcissant, dans certains des domaines qu'étudient les sciences. C'est ainsi 
que d'un côté l'intelligence artificielle, de l'autre Popper, prenant pour objet d'étude respectivement la cognition et l'entreprise scientifique, expliquent toute activité de connaissance en fonction de problèmes purement objectifs, antérieurs aux efforts déployés pour les résoudre. De même l'organisme et, pour les adaptationnistes, la Nature elle-même ne feraient que résoudre des problèmes. Enfin, nos activités publiques et privées se ramèneraient également à la résolution de problèmes.

Sous l'effet de la propagation, les concepts subissent des réajustements successifs dans la hiérarchie de leurs traits sémantiques. La première cybernétique des années 1940 conçoit le cerveau comme une machine déductive qui incarne des principes logiques. Le neurone est vu comme un automate fini dont l'état actif ou passif désigne des valeurs «vrai» ou «faux». Les connexions neuronales forment des portes logiques «et», «ou», «non», etc. La science cognitive y voit depuis 1956 un système interprétatif, théâtre de représentations mentales dont la réalité physique se présente sous la forme d'un système de symboles, tandis que les neurosciences le définissent comme ensemble de cellules actives constituées en dispositif de traitement de l'information qui réagit à l'environnement. Les néo-cybernéticiens (F. Varela, 1989, p. 48) y décèlent, pour leur part, une armée de constituants simples, non intelligents (neurones) dont l'interconnexion dense crée les conditions propices à l'émergence de l'intelligence en tant qu'épiphénomène.

Un autre effet de la propagation est l'apparition de définitions et de désignations parallèles pour un même concept, inspirées ou exigées par les différentes catégories des publics censés s'en servir. On pourrait comparer, par exemple, les définitions rédigées par deux commissions de terminologie pour les termes «informatique», «robotique», «télématique», ou celles du concept «calcul optique» dans des ouvrages spécialisés et des périodiques de vulgarisation où l'on trouve l'expression «calcul lumineux».

L'innovation conceptuelle favorise parfois le recours à la néologie formelle . Ainsi la création du terme "autopoièse" (du grec "autos", soi-même, et "poiein", produire) et des dérivés antonymes «autopoiétique/allopoiétique» pour désigner la notion d'autonomie et d'autoorganisation des systèmes vivants est motivée par la nécessité d'éviter le piège du préconçu:

The introduction of this word... simplified enormously the task of talking about the organization of living without falling into the always gaping trap of not saying anything new because the language does not permit it. We could not escape being immersed in a tradition, but with an adequate language we could orient ourselves differently and, perhaps, from the new perspective generate a new tradition. (H. Maturana et $F$. Varela, 1980, p. XVII)

D'autres concepts encore peuvent recevoir des désignations nouvelles dans une discipline connexe et être transférés tels quels en IA. 
C'est le cas des néologismes «fractal", "amassement", "poussière", «randonnée de Bernoulli» ou «téragone» créés depuis 1970 par le mathématicien français Benoît Mandelbrot, et incorporés récemment au vocabulaire de l'IA néo-connexionniste. Le motif avoué de la dénomination est ici l'efficacité communicative:

C'est par nécessité que mes travaux semblent regorger de néologismes. Même lorsque les idées de base sont anciennes, elles avaient été si peu essentielles qu'on n'avait pas éprouvé le besoin de termes pour les désigner, ou qu'on s'était contenté d'anglicismes ou de termes hâtifs ou lourds ne se prêtant pas aux larges usages que je propose. (B.Mandelbrot, 1984, p. 53)

La définition de l'homme comme «animal symbolique» et l'assimilation du cerveau à un mécanisme calculatoire ont eu des conséquences importantes sur le plan de la dénomination. Une des plus évidentes est le recours à la métaphore anthropomorphique ou à la métonymie pour souligner les similarités fonctionnelles de l'homme et de l'ordinateur. Le cerveau devient un «ordinateur biologique», une «meat machine» ou «wet machine»; l'ordinateur reconnaît les objets à partir de leur "squelette", et toute une série de personnages fictifs prêtent leur nom aux fonctionnalités des machines "pensantes". C'est ainsi que la terminologie des systèmes experts abonde en «démons», «lutins», «dragons", «djinns», «phantômes» et «homoncules». Les noms de métiers servent le même but: «acteur", "évaluateur", "apprenti-programmeur", «superviseur», «diagnosticien», «pédagogue», «strategy judge», «rule sponsor", "tutor» ou «interpreter" sont en fait des programmes ou des routines dont la désignation suit la consigne donnée avec «système expert». La siglaison (S. Pavel, 1988) sert à la remotivation de syntagmes complexes par le recours aux noms propres et aux diminutifs (BORIS, CYRUS, MARGIE, MAIA, NOAH, SAM, SOPHIE), à la métonymie ou à la synecdoque (BRAIN, ESPRIT, PROUST, SAGESSE, SCARABÉE, SNARK).

Ainsi que le montre J. Guillemin-Flescher (1981, pp. 201-228) en comparant la syntaxe des phrases «I heard her laugh at me as I walked away/ Un rire moqueur m'accompagna" et "This system is very popular with passengers/ Ce sytème a reçu un accueil favorable de la clientèle», l'animation des inanimés est plus marquée en anglais qu'en français. On pourrait inférer que le recours à la métaphore anthropomorphique par l'intelligentsIA américaine est motivé par les particularités syntaxiques de l'anglais et qu'en l'adoptant le français subit une influence étrangère.

Le fait que cette préférence dénominationnelle - où l'on attribue à l'inanimé les qualités du vivant pensant - se retrouve aujourd'hui dans des néologismes IA en langues autres que l'anglais s'explique certes, en partie, par le rôle du mimétisme culturel dans l'histoire des civilisations, par le rôle de la traduction dans la dissémination du 
savoir spécialisé et aussi par l'exigence primordiale en technolangue de préserver le sens littéral du texte de départ.

L'explication reste toutefois partielle et partiale tant qu'elle ne tient pas compte d'un autre fait important, à savoir l'universalité de la métaphore anthropomorphique, de l'analogie animé/inanimé et plus généralement de la symétrie d'échelle local/global en tant que procédés de découpage du réel (G. Lakoff et M. Johnson, 1980). L'adoption d'expressions telles que «demon» ou «interpreter" s'explique tant par leur résonance universelle que par leur appartenance au paradigme technoscientifique de l'IA et par la diffusion internationale de l'anglais. Il n'est d'ailleurs pas sans intérêt de remarquer que l'anthropomorphisme métaphorique est également présent dans les contributions originales des chercheurs francophones (ex. le «spécialiste» de $\mathrm{H}$. Wertz, 1985) ou hispanophones (ex. les «coordinateurs» de F. Flores, 1982) et même chez des chercheurs opposés à cette tendance (ex. «computer blindness» et «computer coaching» chez T. Winograd, 1986, ou la notion du langage en tant que «consensual domain" ou «structural coupling" chez H. Maturana, 1970). Les rapports entre la vision du monde propre à une technoscience et l'universalité des moyens linguistiques choisis pour l'exprimer ont une influence non négligeable sur ce que les anthropologues appellent «spread of non-linguistic cultural items». (E. Haugen, 1950, p. 212)

La diffusion de certains effets de langage ne concerne pas que les technolectes. Elle peut atteindre les langues jusque dans leurs registres familiers. En notant la prolifération des locutions néologiques où figure le mot «problème» (Où est le problème? C'est pas mon problème! Il n'y a pas de problème) et qui sont des traductions littérales d'expressions familières en anglais américain (Where's the problem? That's your problem! No problem!), Daniel Andler y détecte «un symptôme d'épidémie culturelle marquée par un positivisme et un pragmatisme caractéristique». (1987, p. 154) Ici encore, bien plus qu'une caractéristique culturelle diffusée par des moyens linguistiques, G. Hottois $(1987$, p. 73 ) voit dans ce pragmatisme un trait éthique intrinsèque de la société technicienne.

Les particularités lexicales de certaines langues peuvent faciliter la diffusion translinguistique de certains effets de langage à l'intérieur d'une communauté technoscientifique. Telle, dans la plupart des langues indo-européennes, l'homonymie des suffixes désignant l'instrument et l'agent. En français, espagnol, italien et allemand, tout au moins, le suffixe -eur/-or/-ore/-er est un des plus grands producteurs de néologismes IA désignant des activités auparavant attribuées à un animé humain (actualisateur, coordinateur, débogueur, démontreur, dépanneur, élagueur, émulateur, extracteur, formaliseur, grapheur, interpréteur, intégrateur, interrogateur, justificateur, programmateur, raisonneur, réconnaisseur, rénovateur, résolveur, restituteur, résumeur, 
schématiseur, simulateur, etc.). On peut certes se demander si ce modèle de dérivation aurait été aussi productif si un modèle équivalent n'avait pas été exploité d'abord en anglais, mais on ne peut douter pour autant de son appartenance aux paradigmes dérivationnels du français, de l'espagnol, de l'italien ou de l'allemand.

\section{Emprunt, adaptation, innovation}

La notion d'emprunt englobe dans ce contexte une partie considérable de la terminologie technoscientifique, de la simple importation pratiquée, par exemple, en japonais (R. A. Miller, 1967) où 70\% de la terminologie de l'informatique est obtenue par la transcription de termes anglais en kana - en passant par les traductions littérales préférées en chinois (V. Alleton, 1986), et jusqu'aux substitutions ingénieuses de mots d'origine étrangère, comme il arrive parfois en français (canal banalisé/citizen's band, bogue/bug ou boule/bulldozer). On peut l'approcher en adoptant le point de vue de J. Humbley (1974, pp. 46-70) selon lequel tout élément d'une langue $A$ constitue un «emprunt lexical» dans une langue B lorsque celle-ci suit le modèle de formation de la langue de départ (décompositionnel, monotonique, planning, intractable), et un «emprunt sémantique» lorsque le modèle étranger n'est pas suivi, soit qu'on lui préfère un modèle autochtone (adaptativité/adaptivity), soit qu'on élargisse le sens d'un mot déjà véhiculé en $B$ (réaliser, falsifier, ou «épervier» au sens de "partisan de la guerre»).

L'emprunt y est défini en tant que passage d'un élément (d'expression, de contenu, ou des deux) d'une langue à une autre, intégré au «processus d'adoption de mots nouveaux ou néologie» (p. 5l) et analysé selon une typologie exhaustive qui tient compte de ses manifestations à tous les niveaux de la langue emprunteuse - phonétique (meeting), graphie (shampooing), morpho-syntaxe (insatisfiabilité/insatisfiability, recherche meilleur d'abord/best-first search) ou sémantique (stratégie/strategy au sens de «algorithme qui contrôle l'application des règles d'inférence»). Cette typologie englobe les fauxemprunts (forme étrangère et contenu autochtone différent ou inexistant dans la langue de départ, tels que footing et tennisman) aussi bien que les emprunts internes (revivification d'archaïsmes, changements de registre et de dialecte, de sociolecte ou de technolecte) dans la même langue.

On peut s'attendre à ce qu'une telle définition ne fasse pas l'unanimité des lexicographes. Certains lui préféreront la définition restreinte aux formes lexicales exogènes; d'autres trouveront peut-être qu'elle empiète sur la création lexicale, qu'elle ne distingue pas nettement l'adoption de l'innovation, ou qu'elle rappelle des réticences exprimées par Haugen à l'endroit du terme «emprunt»: 
The metaphor implied is certainly absurd, since the borrowing takes place without the lender's consent or even awareness, and the borrower is under no obligation to repay the loan. One might as well call it stealing, were it not that the owner is deprived of nothing and feels no urge to recover his goods. The process might be called adoption, for the speaker does adopt elements from another language into his own. But what could one call a word that has been adopted - an adoptee? Anthropologists speak of «diffusion» in connection with a similar process in the spread of non-linguistic cultural items. We might well speak of linguistic diffusion, though this would suggest the spread of the language itself rather than of elements of it... The heart of our definition of borrowing is then the attempted reproduction in one language of patterns previously found in another... The term reproduction does not imply that a mechanical imitation has taken place; on the contrary, the nature of reproduction may differ very widely from the original... (E. Haugen, 1950, pp. 211-212)

Il n'en reste pas moins que, en intelligence artificielle comme en informatique, on peut historiquement avancer que la terminologie a été élaborée en anglo-américain et que les versions dans les autres langues sont des adaptations plus ou moins fidèles. Appliquée à l'analyse du vocabulaire français et allemand de l'informatique (J. Humbley, 1988, pp. 85-148), cette approche s'avère d'ailleurs particulièrement bien adaptée à une évaluation rigoureuse du degré de pénétration des éléments exogènes et des manifestations concrètes de la créativité lexicale en tant que faculté d'adaptation de la langue d'accueil.

L'évolution du néologisme d'emprunt mérite toutefois une attention particulière car c'est dans cette évolution qu'on peut saisir le passage de la créativité adaptatrice à la créativité innovatrice. En intelligence artificielle, on a pu constater que des emprunts directs tels que recherche «depth-first» ou stratégie «least-commitment» sont spontanément remplacés d'abord par des calques (recherche profondeurd'abord, stratégie de moindre engagement) et ensuite par des constructions propres à la syntaxe française (recherche en profondeur, stratégie d'implication minimale). Des hybrides et des traductions littérales cèdent graduellement la place à des créations plus originales: «insatisfiabilité» s'efface devant «insatisfaisabilité», «neurone formel» (formal neuron), synonyme de «neuronoïde» (neuronoid) est remplacé par «neuromime» construit par analogie à «biomime» et servant de base de dérivation à «neuromimétique» sur le modèle de «biomimétique».

Loin de conclure que les traducteurs sont seuls responsables de la prolifération des traductions littérales ou que le mérite du redressement de la situation revient systématiquement aux spécialistes - auteurs d'ouvrages dans la langue d'accueil, force nous est de constater que la créativité lexicale des traducteurs concurrence avantageusement celle des spécialistes: en intelligence artificielle, c'est dans les traductions d'ouvrages anglo-américains qu'on a pu relever nombre de créations 
raisonnées telles que Chose Soyable/Be-Able Thing, CS-ité/BAT-itude, labilité/slipperiness, quasi-instance/near miss, stratégie des unités/unitpreference strategy, qui remplacent des emprunts directs et des traductions littérales spontanément mis en circulation par des auteurs français.

Ces quelques observations incitent à penser qu'avant de tirer leurs conclusions, les néologues chercheront l'innovation lexicale dans les échanges linguistiques quotidiens entre spécialistes et dans les discours de vulgarisation autant que dans les contributions théoriques et pratiques originales des chercheurs francophones, hispanophones, etc., et qu'ils en compareront les procédés terminogènes à ceux qui régissent la naturalisation des néologismes d'emprunt. Pareille démarche sociolinguistique fournirait des informations pertinentes sur le fonctionnement de la néologie technoscientifique.

Étudiée par la rhétorique du $\mathrm{XIX}^{\mathrm{e}}$ siècle en tant que produit de la nécessité, de l'imagination, de l'esprit et de la passion, et par la linguistique du $\mathrm{XX}^{\mathrm{e}}$ siècle en tant que génération d'unités significatives à partir d'ensembles d'éléments, de règles et de contraintes combinatoires, l'activité néologique pourrait être envisagée avec profit dans la perspective ouverte en sciences cognitives par l'analyse formelle de la créativité artistique et de la découverte technoscientifique en tant que mécanismes simulables informatiquement, et en psychologie sociale par la représentation de l'innovation en tant que motivation du comportement, activité sociale de signalisation/interprétation symbolique et restructuration spatio-temporelle de l'univers discursif.

\section{Références}

Alleton, V. (1986). Dictionary of Loan Words and Hybrid Words in Chinese. Shanghai, 1984; compte-rendu dans Cahiers de linguistique d'Asie Orientale, vol. $15, \mathrm{n}^{\circ} 2$, pp. 339-343.

ANDLER, D. (1987). «Problème. Une clé universelle?» In I. Stengers et al. Les Concepts nomades. Paris, Seuil, pp. 119-159.

Cellard, J. (1987). «Paroles dans l'air. Quand les mots racontent l'histoire». Le Débat, ${ }^{\circ}$ 50, pp. 226-230.

Dougnac, F. (1982). «Aspects de la néologie lexicale dans le Journal de la langue françoise (1784-1795)». Linx (Bulletin du centre de recherches de Paris-Nanterre), vol. 2, $\mathrm{n}^{\circ}$ 7, pp. 7-53.

FLORES, F. (1982). Management and Communication in the Office of the Future. San Francisco, Hermenet Inc.

Gullbert, L. (1975). La créativité lexicale. Paris, Larousse. 
GullLemin-Flescher, J. (1981). Syntaxe comparée du français et de l'anglais. Problèmes de traduction. Paris, Ophrys.

HaGege, Cl. (1987). Le Français et les siècles. Paris, Odile Jacob.

HAUGEN, E. (1950). «The Analysis of Linguistic Borrowing». Language, vol. $26, \mathrm{n}^{\circ} 2$, pp. $210-231$.

HOPE, T. E. (1971). Lexical Borrowing in the Romance Languages. 2 vol. Oxford, Basil Blackwell.

HоттоIs, G. (1987). «Technolangues et technophobie philosophique». Penser l'informatique, informatiser la pensée. Mélanges offerts à André Robinet, L. Couloubaritsis et G. Hottois, éd. Éditions de l'Université de Bruxelles, pp. 59-75.

Humbley, J. (1974). "Vers une typologie de l'emprunt linguistique». Cahiers de lexicologie, vol. 25, $\mathrm{n}^{\circ} 2$, pp. 46-70.

Humbley, J. (1988). «Comment le français et l'allemand aménagent la terminologie de l'informatique». La Banque des Mots, numéro spécial, Paris, CILF-CNRS-INaLF, pp.85-148.

LAKOFF, G. et M. Johnson (1980). Metaphors We Live By. Chicago, London, Univ. of Chicago Press.

LENAT, D. (1978). "The Ubiquity of Discovery». Artificial Intelligence, vol. 9, $\mathrm{n}^{\circ} 3$, pp. $257-287$.

Mandelbrot, B. (1984). Les Objets fractals, Paris, Flammarion.

Maturana, H. et F. Varela (1980). Autopoiesis and Cognition: The Realization of the Living. Dordrecht, Reidel.

Miller, R. A. (1967). The Japanese Language. Chicago, London, The University of Chicago Press.

PAVEL, S. (1988). «Siglaison et créativité lexicale en intelligence artificielle». L'Actualité terminologique, vol. $21, \mathrm{n}^{\circ} 4$, pp. 1-4.

Revel, J.-F. (1988). La connaissance inutile. Paris, Grasset.

REY, A. (1976). «Néologisme: un pseudo-concept?». Cahiers de lexicologie, vol. $28, \mathrm{n}^{\circ} 1, \mathrm{pp} .3-17$.

Rey, A. et J. Rey-Debove (1988). Le Petit Robert. Dictionnaire alphabétique et analogique de la langue française. Vol.1, Paris, Le Robert.

Rey-Debove, J. (1978). Le Métalangage: étude linguistique du discours sur le langage. Paris, Le Robert.

STENGERs, I. et coll. (1987). D'une science à l'autre, des concepts nomades. Paris, Seuil.

ThIEle, J. (1987). La formation des mots en français moderne. Traduit et adapté de l'allemand par André Clas. Presses de l'Université de Montréal.

Turner, J. (1988). A Theory of Social Interaction. Stanford, Calif., Stanford University Press. 
VARela, F. (1989). Connaître. Les sciences cognitives. Tendances et perspectives. Paris, Seuil.

WERTZ, H. (1985). Intelligence artificielle; application à l'analyse de programmes. Paris, Masson.

Winograd, T. et F. Flores (1986). Understanding Computers and Cognition: a New Foundation for Design. Norwood, New Jersey, Ablex Publ. Corp. 